\title{
The effects of feeding mixed tocopherol oil on whole-blood respiratory burst and neutrophil immunometabolic-related gene expression in lactating dairy cows
}

\author{
Y. Qu, ${ }^{*}$ T. H. Elsasser, ${ }^{*}$ S. Kahl, ${ }^{*}$ M. Garcia,† C. M. Scholte,‡ E. E. Connor,§ G. F. Schroeder,\# \\ and K. M. Moyes $¥ 1$ \\ *Agricultural Research Service, Animal Biosciences and Biotechnology Laboratory, USDA, Beltsville, MD 20705 \\ †Department of Animal Sciences and Industry, Kansas State University, Manhattan 66506 \\ ‡Department of Animal and Avian Sciences, University of Maryland, College Park 20472 \\ §Agricultural Research Service, Animal Genomics and Improvement Laboratory, USDA, Beltsville, MD 20705 \\ \#Cargill Inc., Elk River, MN 55330
}

\begin{abstract}
The 4 major tocopherol isoforms differ in their biochemical reactivity and cellular effects due to basic chemical structural differences. Alpha-tocopherol has been well studied regarding effects on bovine polymorphonuclear leukocyte (PMN) function and its involvement in respiratory burst. However, no studies to date have identified the effects of supplementing a mixed tocopherol oil (Tmix) particularly enriched in non- $\alpha$ tocopherol isoforms (i.e., $\gamma$ - and $\delta$-isoforms) on fundamental immunometabolic changes in dairy cows. Therefore, the objectives of this study were to determine whether short-term feeding of vegetable oil-derived Tmix alters specific biomarkers of metabolism, whole-blood leukocyte populations, respiratory burst, immunometabolic-related gene expression of PMN, or gene expression of isolated PMN when challenged with lipopolysaccharides (LPS). Clinically healthy multiparous lactating Holstein cows $(\mathrm{n}=12 ; 179 \pm$ $17 \mathrm{~d}$ in milk, $40.65 \pm 3.68 \mathrm{~kg}$ of milk yield) were fed Tmix $(620 \mathrm{~g} / \mathrm{d})$ for 7 consecutive days. Jugular blood (EDTA anticoagulant) was collected from all cows on d 0 before treatment initiation and again on d 7 after Tmix feeding. Total stimulated respiratory burst activity (RBA) and leukocyte populations were assessed in whole blood, and tocopherol isoform concentrations, metabolites, and hormones were measured in plasma. For gene expression analysis, isolated PMN from cows before and after Tmix feeding were incubated with LPS at a final concentration of either 0.0 or $1.5 \mu \mathrm{g} / \mathrm{mL}$. Feeding of Tmix for $7 \mathrm{~d}$ increased the concentrations of $\alpha$ - and $\gamma$-tocopherol. The Tmix did not alter plasma insulin but decreased cholesterol. The Tmix did not alter whole-blood RBA or the leukocyte populations. The
\end{abstract}

Received September 26, 2017.

Accepted January 11, 2018.

${ }^{1}$ Corresponding author: kmoyes@umd.edu
LPS challenge increased the expression of proinflammatory genes TNFA and IL6. However, Tmix treatment did not alter the patterns of LPS-affected expression of genes (e.g., TNFA, ITGB2, PPARA, and RXRA) associated with the immune or metabolic response. In conclusion, short-term feeding of Tmix may have no negative effect on animal health as Tmix increased $\alpha$ - and $\gamma$-tocopherol concentrations in blood and did not impair whole-blood RBA or alter leukocyte populations. The data provide further support that the $\alpha$ - and $\gamma$-tocopherol isoforms do not interfere with normal immune or metabolic function.

Key words: Holstein cow, mid lactation, tocopherol, health, neutrophil

\section{INTRODUCTION}

Bovine mastitis (i.e., an inflammation of the mammary gland) is usually associated with the invasion of a bacterial pathogen (Gruet et al., 2001). During mastitis, circulating PMN are recruited to the infection site and play an essential role in controlling the duration and severity of the infection-recovery process (Paape et al., 2003; Lauzon et al., 2005). However, PMN can have detrimental effects on the mammary tissue during the inflammatory response. Reasons could be host cell mammary gland damage (mainly via lipid peroxidation) associated with the overproduction of proinflammatory cytokines [i.e., tumor necrosis factor (TNF) $\alpha$; Persson et al., 1993] and reactive oxygen species (ROS; Sordillo et al., 2009). These proinflammatory factors are acknowledged to contribute significantly to collateral metabolic disorders such as ketosis and milk fever or inflammation in other organs (Blum et al., 2000; Wellnitz and Bruckmaier, 2012; Sordillo, 2016).

Nutritional supplements of vitamin E have been studied with regard to mitigating the negative consequences of the inflammatory response in cows and calves (Barrett et al., 1997; Higuchi et al., 2013; Singh et al., 
2013). Potential mechanisms of the beneficial effects of vitamin E during inflammation include (1) helping to control lipid peroxidation of host tissues mainly associated with infiltrating PMN, (2) controlling lipid peroxidation-induced inflammation, and (3) protecting the integrity of the membrane structure of PMN via alleviating lipid peroxidation during inflammation (Chew, 1995). Alpha-tocopherol (i.e., the most biologically active isoform of vitamin E) has been studied for its ability to improve PMN chemotaxis (Luostarinen et al., 1991) and enhance the expression of plasminogen activator urokinase receptor gene (PLAUR; Pinotti et al., 2003). In addition, $\alpha$-tocopherol has been studied as a means of improving phagocytosis of PMN in cattle (Hogan et al., 1992) and modulating the expression of proinflammatory genes in PMN by controlling lipid peroxidation (i.e., oxidative stress) responses (Sordillo et al., 2009). Most vitamin E research has been conducted during the transition period (Qu et al., 2013; Pilotto et al., 2016), a high-risk period for disease (Drackley et al., 2006) when most cows experience a natural depletion of vitamin $\mathrm{E}$ (i.e., $\alpha$-tocopherol). This depletion can increase susceptibility to disease (Pinotti et al., 2003; Qu et al., 2013, 2014). Much less information is available on the mitigating effects of vitamin E during mid lactation. For the current study, dairy cows in mid lactation were used to characterize the host response to feeding tocopherols to reduce the potential cofounding complications of the high-risk transition period.

Together, the $\alpha-, \beta-, \gamma-$, and $\delta$-tocopherol isoforms constitute the naturally occurring mixed tocopherol matrix (Spears and Weiss, 2008; Weiss and St-Pierre, 2009; Politis et al., 2012). In dairy cattle, $\alpha$-tocopherol, the major isoform used in diet supplementation, has been studied more than any other isoform. Furthermore, because so much emphasis has been placed on the biological attributes of $\alpha$-tocopherol, a major role for the other isoforms has been related to their conversion to the $\alpha$-isoform (Netscher et al., 2007). What is recognized now that was not apparent at the time when $\alpha$-tocopherol was first added as a supplement to the diet is that these non- $\alpha$ isoforms have a more diverse biochemical pedigree with their own unique healthful properties distinct from those attributable to $\alpha$-tocopherol. For example, $\gamma$-tocopherol is recognized as distinct from $\alpha$-tocopherol as an anti-inflammatory intervention where $\gamma$-tocopherol possesses cyclooxygenase activity and has a higher affinity to trap lipophilic electrophiles, many of which are derived from superoxide anion and nitric oxide during an inflammatory event within the membrane (Groeger and Freeman, 2010). In addition, mixed tocopherol is a natural oil extracted from several plant sources such as soybean and could have a positive effect on organic dairy practices. However, the effect of feeding additional mixed tocopherol isoforms on animal health has not been fully elucidated. It is becoming clearer that $\gamma$ - and $\delta$-tocopherols have overall health benefits additive and complementary to $\alpha$-tocopherol (Zempleni et al., 2013). Therefore, the objectives of this study were to investigate how short-term feeding of vegetable oil-derived mixed tocopherol oil (Tmix) supplement (with high levels of $\gamma$ - and $\delta$-isoforms) might alter specific biomarkers of metabolism, wholeblood leukocyte populations and respiratory burst, and PMN immunometabolic-related gene expression. The hypotheses are that Tmix $(\alpha-, \beta-, \gamma-, \delta$-tocopherol) will not detrimentally alter animal health as determined via biomarkers of metabolism, leukocyte population, and respiratory burst status and that Tmix may differentially affect the expression of proinflammatory genes in circulating PMN.

\section{MATERIALS AND METHODS}

All procedures involving the use of live animals were approved in accordance with the regulations and guidelines set forth by the USDA Beltsville Animal Care and Use Committee (no. 15-009).

\section{Animals and Treatments}

Twelve multiparous Holstein cows in mid lactation $(179 \pm 17 \mathrm{DIM} ; 40.65 \pm 3.68 \mathrm{~kg} / \mathrm{d}$ milk yield) were used for this study. Due to the tiestall space limitation, the experiment was conducted in 3 blocks of 4 cows each (randomly picked from a total of 12 cows). Cows did not display clinical signs of disease and had an average composite milk SCC $<100,000$ cells/mL before enrollment in the study. Within each block, cows ( $\mathrm{n}=$ 4) were housed and fed in tiestalls (each with its own feed bunk), had free access to water, and were milked twice daily at 0600 and $1800 \mathrm{~h}$. Cows were fed a TMR $(22.7 \mathrm{~kg} \pm 1.3 \mathrm{~kg} / \mathrm{d})$ ad libitum (Table 1) formulated to meet NRC (2001) requirements $\left(\mathrm{NE}_{\mathrm{L}}=1.5 \mathrm{Mcal} / \mathrm{kg}\right.$ of $\mathrm{DM} ; \mathrm{CP}=17.03 \%$ of $\mathrm{DM}$ ) for lactating dairy cows averaging $90 \mathrm{DIM}$ and producing $40 \mathrm{~kg}$ of milk/d with a BW and BCS of $598 \mathrm{~kg}$ and 3.0 (on a 5-point scale; Ferguson et al., 1994), respectively. Cows were fed twice per day at 0700 and $1400 \mathrm{~h}$ with the diet portions equally split between the 2 feedings (total of $22.7 \pm 1.3$ $\mathrm{kg}$ of $\mathrm{TMR} / \mathrm{d}$ ). An initial blood sample was collected on $\mathrm{d} 0$ before the morning feeding to represent the basal diet without Tmix supplementation (absence: -Tmix). All cows then received their morning feed top-dressed with the Tmix $(620 \mathrm{~g} / \mathrm{d})$ for 7 consecutive days. Blood sampling was repeated on d 7 after Tmix supplementation to represent the diet with Tmix supplementation (presence: +Tmix). The preparation of the Tmix for 
top-dressing was previously described (Elsasser et al., 2013). In brief, a concentrated mixed tocopherol oil (soybean extract, $300 \mathrm{~g}$, Cargill Innovation Center, Velddriel, the Netherlands; isoform composition: $9 \% \alpha-$, $1 \% \beta$-, $24 \% \delta$-, and $62 \% \gamma$-tocopherol) was mixed into a commercial molasses-sweetened calf grower diet (22.68 kg; Farmers Cooperative Association Inc., Frederick, $\mathrm{MD})$ as the carrier and to improve the likelihood that all the supplement was consumed at the top-dressing. By analysis, each individual cow received from the basal TMR 1,442 $\pm 86 \mathrm{mg}$ of $\alpha$-tocopherol, nondetectable $\beta$-tocopherol, $355 \pm 5 \mathrm{mg}$ of $\gamma$-tocopherol, and 89 $\pm 21 \mathrm{mg}$ of $\delta$-tocopherol daily. From the supplemental Tmix, each individual cow received additionally $740 \mathrm{mg}$ of $\alpha$-tocopherol, $80 \mathrm{mg}$ of $\beta$-tocopherol, $5,080 \mathrm{mg}$ of $\gamma$-tocopherol, and 1,970 $\mathrm{mg}$ of $\delta$-tocopherol daily. The amount of Tmix was the same as previously described in a published study using the mixed tocopherols in beef heifers (Elsasser et al., 2013). The selected amount of Tmix is consistent with the levels of $\alpha$-tocopherol used in dairy cows previously (Politis et al., 1996; Weiss et al., 1997) and extends the findings with the inclusion of the non- $\alpha$ tocopherol isoforms.

\section{Blood Collection and Leukocyte Population}

Whole blood $(\sim 40 \mathrm{~mL})$ was collected into EDTA tubes (Becton Dickinson Vacutainer System, Franklin Lakes, NJ) for analysis of respiratory burst activity. Blood collected in Vacutainer tubes containing EDTA was analyzed for leukocyte population within $1 \mathrm{~h}$ of collection using a ProCyte Dx hematology analyzer (Idexx Laboratories Inc., Westbrook, ME). Plasma for analysis of metabolites, insulin, and tocopherol concentration was collected after centrifugation at 1,300 $\times g$ for $15 \mathrm{~min}$ at $4^{\circ} \mathrm{C}$ and stored in $1.5-\mathrm{mL}$ tubes at $-20^{\circ} \mathrm{C}$ for future analysis. For gene expression analysis, jugular blood [ $\sim 200 \mathrm{~mL}$; i.e., sampling on d 0 (-Tmix) and $\mathrm{d} 7$ (+Tmix)] was collected from each cow into 15 -mL tubes containing $1 \mathrm{~mL}$ of acid-citrate dextrose (Thermo Fisher Scientific Inc., Pittsburgh, PA), gently inverted to mix, and placed on ice. Blood samples were processed for PMN isolation within $1 \mathrm{~h}$ after collection as described below.

\section{Whole-Blood Respiratory Burst by Chemiluminescence}

Luminol-dependent chemiluminescence procedures followed those as previously described (Mehrzad et al., 2002) with minor modifications. Phorbol 12-myristate 13-acetate (PMA) was chosen for incubation with blood instead of LPS to mimic the production of ROS by PMN during phagocytosis (Tian et al., 2005)
Table 1. Ingredient and nutrient composition (DM basis) of the TMR ${ }^{1}$ in the experimental diets fed for $7 \mathrm{~d}$

Item, $\%$ of dietary DM

unless noted otherwise

Amount

\begin{tabular}{lr}
\hline Ingredient & \\
Corn silage & 31.0 \\
Alfalfa-wheat silage & 8.0 \\
Cottonseed, whole & 4.5 \\
Citrus pulp, dehydrated & 4.5 \\
Sugar blend & 3.9 \\
Alfalfa hay & 3.6 \\
Grass hay & 3.6 \\
Grain mix & 39.1 \\
Nutrient content & \\
DM & 52.2 \\
NDF & 28.1 \\
ADF & 18.4 \\
CP & 17.0 \\
Ether extract & 6.0 \\
NFC & 43.4 \\
Calcium & 0.9 \\
Phosphorus & 0.3 \\
Magnesium & 0.5 \\
Potassium & 1.2 \\
ME, Mcal/kg of DM & 2.5 \\
Vitamin A, kIU/kg of DM & 19.7 \\
Vitamin D, kIU/kg of DM & 4.9 \\
Vitamin E, IU/kg of DM & 16.4 \\
\hline
\end{tabular}

${ }^{1}$ The TMR (52.2\% DM) contained corn silage (31.0\%), alfalfa-wheat silage $(8.0 \%)$, alfalfa hay $(3.6 \%)$, grass hay $(3.6 \%)$, whole cottonseed (4.5\%), dehydrated citrus pulp (4.5\%), sugar blend (3.9\%), and lactation grain mix $(39.1 \%)$. On a DM basis, the ration contained $17.0 \%$ CP, $18.4 \%$ ADF, 28.1\% NDF, 3.2\% lignin, $6.0 \%$ ether extract, $0.9 \%$ calcium, $0.3 \%$ phosphorus, $1.2 \%$ potassium, $0.5 \%$ magnesium, and 2.49 Mcal of ME $/ \mathrm{kg}$.

${ }^{2}$ The sugar blend $(3.9 \% \mathrm{DM})$ contained CP $(1.0 \%)$, crude fat $(0.1 \%)$, crude fiber $(0.1 \%), \operatorname{ADF}(0.1 \%)$, calcium $(0.75 \%)$, phosphorus $(0.1 \%)$, cobalt $(8 \mathrm{mg} / \mathrm{kg})$, copper $(138 \mathrm{mg} / \mathrm{kg})$, manganese $(345 \mathrm{mg} / \mathrm{kg})$, zinc (690 mg/ $\mathrm{kg})$, selenium $(8.27 \mathrm{mg} / \mathrm{kg})$, vitamin A $(8,377 \mathrm{IU} / \mathrm{kg})$, vitamin $\mathrm{D}_{3}(13,888 \mathrm{IU} / \mathrm{kg})$, vitamin $\mathrm{E}(277 \mathrm{IU} / \mathrm{kg})$, total sugar as invert (33.0\%), DM (60.0\%), and moisture (40\%).

${ }^{3}$ The grain mix $(39.1 \% \mathrm{DM})$ contained finely ground corn $(92.0 \%)$, AminoPlus (1.24\%), soybean meal $48 \%(1.24 \%)$, whole roasted soybean $(1.55 \%)$, soybean hulls $(1.17 \%)$, Megalac $(0.75 \%)$, LysAAmet $(0.39 \%)$, Optigen $(0.42 \%)$, limestone $(0.34 \%)$, white salt $(0.21 \%)$, magnesium oxide $(0.17 \%)$, USDA custom dairy mix no. $4(0.15 \%)$, potassium-magnesium sulfate $(0.11 \%)$, sodium bicarbonate $(0.08 \%)$, urea $45 \%$ (0.04\%), MFP Novus (0.04\%), Mepron M85 (0.02\%), Rumensin $90(0.003 \%)$, Rovimis H-2 (Biotin) (0.001\%), and Zinpro-4-Plex-C $(0.001 \%)$ (Westway Feed Products LLC, New Orleans, LA).

because, unlike LPS, PMA does not require a cell surface receptor (e.g., toll-like receptor-4) or LPS binding protein to stimulate PMN oxidative burst, which might cause high analysis variance (Akira and Hemmi, 2003). The use of whole blood was preferred instead of isolated PMN to avoid potential cell activation and ROS production. Phagocytes such as PMN and monocytes can produce ROS after PMA challenge (Lieberman et al., 1996). However, because the number of PMN was 5 times greater than that of monocytes, the major contributor to ROS production would be PMN (Paape et al., 2003). Preliminary experiments were conducted 
to optimize the incubation conditions, concentration of PMA, and time of incubation response recording needed to obtain repeatable and valid burst activity. Briefly, for each sample test, a set of 2 tubes was filled with $500 \mu \mathrm{L}$ of whole blood and preincubated for 10 min at $37^{\circ} \mathrm{C}$ in an aluminum heating block. Then, 20 $\mu \mathrm{L}$ of working solution of PMA (i.e., stimulant) or water (i.e., nonsimulant) was added to achieve a final concentration of 0.8 or $0.0 \mu \mathrm{g} / \mathrm{mL}$. After incubation for $15 \mathrm{~min}$ at $37^{\circ} \mathrm{C}, 40-\mu \mathrm{L}$ aliquots of blood were mixed with $1 \mathrm{~mL}$ of minimal assay buffer containing luminol $(250 \mu M)$. All assay tubes were incubated for an additional 10 min at $37^{\circ} \mathrm{C}$ and transferred to the chemiluminometer (Autolumat LB 953; E. G. \& G. Berthold, Wildbad, Germany). Chemiluminescence generated in each tube was estimated continuously for $20 \mathrm{~min}$, and measurements of light emission were recorded every 60 $\mathrm{s}(\mathrm{n}=20$ reads/tube). For each sample, mean values of the 2 tubes were used for further data processing. The respiratory burst activity was expressed in relative chemiluminescence units.

\section{Blood Tocopherol Isoform Analysis}

For plasma, $300 \mu \mathrm{L}$ was mixed with $300 \mu \mathrm{L}$ of water containing EDTA $(10 \mathrm{mg} / \mathrm{L})$ and ascorbic acid (100 $\mathrm{mg} / \mathrm{L})$. Samples were precipitated with $600 \mu \mathrm{L}$ of ethanol containing tocopherol (i.e., $\alpha-, \beta-, \delta$-, and $\gamma$-isoforms) as the internal standard and extracted 2 times with $2 \mathrm{~mL}$ of hexane. The combined hexane extracts were vacuum dried and reconstituted in $100 \mu \mathrm{L}$ of hexane. Twenty microliters of prepared solutions were injected into the HPLC system. Separation was performed using the ThermoSeparations Products HPLC system (Thermo Fisher Scientific Inc.) with a Chromegabond Diol column (ES Industries, West Berlin, NJ; $3 \mu \mathrm{m}, 150$ $\times 4 \mathrm{~mm}$ ) equipped with an FL3000 fluorescence detector and a UV2000 programmable UV/visible detector. The mobile phase was $4 \%$ dioxane in hexane pumped at $1.0 \mathrm{~mL} / \mathrm{min}$ and a run time of $20 \mathrm{~min}$. The UV detector was set at $269 \mathrm{~nm}$, and the fluorescence detector was set at 290-nm excitations and 340-nm emissions.

\section{Analysis of Plasma Metabolites and Insulin}

Plasma from all enrolled cows was analyzed for concentrations of nonesterified fatty acids (NEFA), cholesterol, and glucose to further confirm that cows were metabolically healthy. All measures were run on a single 96-well plate following the manufacturer's instructions. Plasma NEFA was analyzed using a commercially available kit (NEFA-HR kit; Wako Diagnostics Inc., Richmond, VA). The intra-assay coefficient of variation $(\mathbf{C V})$ was $4.6 \%$. The plasma cholesterol assay was analyzed using the Liquicolor method from a commercially available kit (Stanbio Laboratories, Boerne, TX). The intra-assay CV was $4.6 \%$ and interassay CV was $6.4 \%$. Plasma glucose was analyzed by a glucose oxidase methodology using a commercially available kit (Stanbio Laboratories). The intra-assay CV was $2.4 \%$. Plasma insulin was analyzed using a solid-phase coated ELISA kit (Thermo Fisher Scientific Inc.). The intraassay CV was $1.8 \%$.

\section{Isolation of Bovine PMN}

Blood PMN were isolated following the previous procedure described by Garcia et al. (2015a). Cell viability was determined using the trypan blue $(0.1 \%$, Bio-Rad Laboratories Inc., Hercules, CA) exclusion method (Freshney, 1987). The average viability was $96 \% \pm$ $1.34 \%$. Differential cell counts of isolated cells were determined microscopically on cytospin preparations using a commercially available hematology staining kit (Hema-Fast 3-Step hematology staining kit; Thermo Fisher Scientific Inc.). The average cell differential was $91 \pm 1.10 \%$ PMN compared with red blood cells.

\section{PMN Treatments and Incubation}

Cell suspensions were diluted to desired concentrations of $4 \times 10^{6}$ cells $/ \mathrm{mL}$ (final volume $2.0 \mathrm{~mL}$ available) using Roswell Park Memorial Institute 1640 medium (RPMI) without glutamine (Sigma-Aldrich Inc., St. Louis, MO) and containing $5 \%$ heat-inactivated fetal bovine serum (Sigma-Aldrich Inc.). Procedures followed those described by Garcia et al. (2016) with minor modifications. Two hundred microliters of PBS containing $1.5 \mu \mathrm{g} / \mathrm{mL}$ of LPS (Escherichia coli 055: B5, Sigma-Aldrich Inc.) or without LPS (control) was mixed with $800 \mu \mathrm{L}$ of RPMI containing $4 \times 10^{6}$ cells/ $\mathrm{mL}$ of isolated PMN cell suspension in 24-well cell culture incubation plates. The cell culture plates were incubated for $120 \mathrm{~min}$ at $37^{\circ} \mathrm{C}, 95 \%$ relative humidity, and $5 \% \mathrm{CO}_{2}$. Following incubation, $1.0 \mathrm{~mL}$ of cell suspension was transferred to 2.0-mL RNase-free tubes (Thermo Fisher Scientific Inc.) and centrifuged (1,000 $\times g$ at $4^{\circ} \mathrm{C}$ for $2 \mathrm{~min}$ ). One milliliter of Trizol (Thermo Fisher Scientific Inc.) was added to the cell pellet and vigorously pipetted up and down to lyse the cells completely for the quantitative reverse-transcription PCR analysis described below.

\section{RNA Isolation, cDNA Synthesis, and Quantitative Reverse-Transcription PCR}

All procedures follow those previously described by Garcia et al. (2016) with minor modifications. Briefly, 
total RNA ( $\mathrm{n}=6 /$ treatment; 4 treatments total) was extracted using the Trizol (Thermo Fisher Scientific Inc.) and EGA-EZNA total RNA kit (Omega Bio-Tek Inc., Norcross, GA) according to the manufacturer's instructions. Genomic DNA was removed with DNAase I enzyme (Bio-Rad Laboratories Inc.). The RNA concentration was measured with the NanoVue Plus (GE Healthcare, Piscataway, NJ). The purity of extracted RNA was calculated by absorbance ratio of $260 / 280$ measured using the NanoVue Plus with an average of $1.74 \pm 0.39$ (mean \pm SD). The integrity of RNA was measured by the RNA quality indicator number, with an average of $8.59 \pm 1.20$, using an Experion bioanalyzer (Bio-Rad Laboratories Inc.). The cDNA was synthesized with $100 \mathrm{ng}$ of RNA per reaction $(20 \mu \mathrm{L})$ using the iScript cDNA synthesis kit (Bio-Rad Laboratories Inc.). Reactions contained $4 \mu \mathrm{L}$ of $5 \times$ reaction mix [blend of oligo (dT) and random hexamer primers] optimized for production of targets $<1 \mathrm{~kb}$ in length, $1 \mu \mathrm{L}$ of reverse transcriptase $\left(\mathrm{RNase} \mathrm{H}^{+}\right)$, and variable volumes of RNA templates to achieve $100 \mathrm{ng}$ and nuclease-free water. The reaction was performed in a T100 Thermal Cycler (Bio-Rad Laboratories Inc.) with a temperature program of $25^{\circ} \mathrm{C}$ for $5 \mathrm{~min}, 42^{\circ} \mathrm{C}$ for 30 min, and $85^{\circ} \mathrm{C}$ for 5 min.

Primer selection and efficiency curves were made following procedures described by Garcia et al. (2015b). The GenBank accession number, gene symbol, hybridization position, sequence, amplicon size, and references for primers used can be found in Supplemental Table S1 (https://doi.org/10.3168/jds.2017-13902). Briefly, primers were selected from previous publications except for $N C F 1$ and $N C F 2$, which were designed using Primer Express 3.0.1 (Fisherbrand, Thermo Fisher Scientific Inc.) with an intended minimum amplicon size of $80 \mathrm{bp}$ (amplicons of 100 to $120 \mathrm{bp}$ were of priority, if possible) and limited $3^{\prime} \mathrm{G}+\mathrm{C}$ percentage. Candidate primers were screened to verify proper sequence with the most updated FASTA sequence for corresponding accession number for Bos taurus with prefix NM_ (messenger RNA), preferentially. Lyophilized primers $(\sim 25$-nmol DNA oligo, with a standard desalting purification method) were obtained from Integrated DNA Technologies (Coralville, IA). Primers were reconstituted with molecular-grade water (G-Biosciences, St. Louis, $\mathrm{MO}$ ) to a concentration of $100 \mu \mathrm{M}$, then diluted to a working primer concentration of $10 \mu M$. The amplification efficiency of primers was verified by a 6-point serial dilution (1:4). Efficiency values ranged from 0.90 to 1.18 , with an average value of $0.99 \pm 0.09$ and an average coefficient of determination of $0.99 \pm 0.001$ (Supplemental Table S2; https://doi.org/10.3168/jds .2017-13902).
Quantitative PCR reactions were performed using SYBR green dye (PerfeCta SYBR Green fast mix; Quanta Biosciences Inc., Gaithersburg, MD). Briefly, $4 \mu \mathrm{L}$ of cDNA template (500 pg of RNA per reaction; diluted from the starting cDNA containing $100 \mathrm{ng}$ of RNA) were combined with $5 \mu \mathrm{L}$ of SYBR Green fast mix, $0.5 \mu \mathrm{L}$ each of forward and reverse primers (500 $\mathrm{n} M$ of each primer per reaction), and $3.4 \mu \mathrm{L}$ of molecular-grade DNase/RNase-free water in a skirted 96-well plate (VWR International, Philadelphia, PA). All samples were run in triplicate and were performed on a single plate per a given gene. The PCR reaction was performed in a CFX96 optical reaction module (Bio-Rad Laboratories Inc.) using the following cycling protocol: initial denaturation at $95^{\circ} \mathrm{C}$ for $30 \mathrm{~s} ; 40 \mathrm{PCR}$ cycles of $95^{\circ} \mathrm{C}$ for $5 \mathrm{~s}$ and $60^{\circ} \mathrm{C}$ for $30 \mathrm{~s}$ for data collection, annealing, and extension; and a final step at $95^{\circ} \mathrm{C}$ for $10 \mathrm{~s}$ before the melting curve. The melting curve step was included to verify the absence of formation of primer dimers and formation of single PCR products using an incremental temperature of $0.5^{\circ} \mathrm{C}$ from $65^{\circ} \mathrm{C}$ to $95^{\circ} \mathrm{C}$ for $5 \mathrm{~s}$. A signal melting curve was expected to indicate the purity of DNA products. The quantitative reverse-transcription PCR results, given as cycle quantification (Cq) values for each transcript, were analyzed using the $2^{-\Delta \Delta \mathrm{Cq}}$ method (Livak and Schmittgen, 2001). Briefly, the Cq of each target gene was calculated and normalized by the geometric mean of the 2 selected reference genes (RPS9, OSBPL2; Garcia et al., $2016)$ to generate $\Delta \mathrm{Cq}$ values. The $\Delta \mathrm{Cq}$ values without transformation were used for statistical analysis. The efficiency of dilution curves and median $\mathrm{Cq}$ values for all tested samples can be found in Supplemental Table S2.

\section{Statistical Analysis}

Changes in whole-blood respiratory burst, leukocyte population, plasma tocopherol isoforms, metabolites, and insulin due to the dietary Tmix treatment were analyzed using PROC MIXED of SAS 9.4 (SAS Institute Inc., Cary, NC) with Tmix treatment (-Tmix vs. +Tmix) and blocks as fixed effects; Tmix treatment was considered repeated on the same cow, which was nested within the block. Cow within the block was used as a random effect. For chemiluminescence, the sum number of PMN and monocytes in whole blood was added in the model as a covariate factor. For gene expression analysis, the data were analyzed using a $2 \times 2$ factorial arrangement with factors including 2 levels of Tmix supplementation (-Tmix vs. + Tmix) and 2 levels of LPS dose (0 or $1.5 \mu \mathrm{g} / \mathrm{mL}$ ). The model included Tmix treatment, LPS challenge, and their interaction 
and blocks as fixed effect. Cow was nested within the block and was used as a random effect.

For all models, the degrees of freedom were estimated with the Kenward-Roger specification in the model statement, and the separation of least squares means and individual comparisons for significant effects were performed using the PDIFF statement in SAS. The data were presented as least squares means and largest standard error of the mean. Statistical differences were declared as significant and highly significant at $P \leq 0.05$ and $P<0.01$, respectively. Trends toward significance are discussed at $0.05<P<0.10$. Due to the repeated measures aspect of the design, with multiple dependent variables, all reported $P$-values were adjusted to control the experimentwise error rate. We used the false discovery rate (Benjamini and Hochberg, 1995) for this adjustment using the P.adjust function in $\mathrm{R}$ ( $\mathrm{R}$ Core Team, 2014). Fold-change values for gene expression analysis were calculated according to Livak and Schmittgen (2001). Briefly, least squares means $(\Delta \mathrm{Cq})$ of a factor of interest (with Tmix or LPS) were subtracted from the least squares means $(\Delta \mathrm{Cq})$ of other referential factor (without Tmix or LPS) to generate the $\Delta \Delta \mathrm{Cq}$ value, which was linearized to a fold-change value with the formula $2^{-\Delta \Delta \mathrm{Cq}}$.

\section{RESULTS}

\section{Tocopherol Isoforms in Bovine Blood With and Without Tmix Supplementation}

The dietary addition of Tmix for a period of $7 \mathrm{~d}$ increased the plasma concentration of $\alpha$-tocopherol (11.19 vs. $14.89 \mu \mathrm{g} / \mathrm{mL} ; P=0.04$; Figure $1 \mathrm{~A}$ ) and the concentration of $\gamma$-tocopherol $(0.63$ vs. $3.05 \mu \mathrm{g} / \mathrm{mL} ; P$ $<0.01$; Figure 1 B).

\section{Plasma Metabolites and Insulin}

Concentrations of plasma metabolites and insulin before and after Tmix supplementation are shown in
A

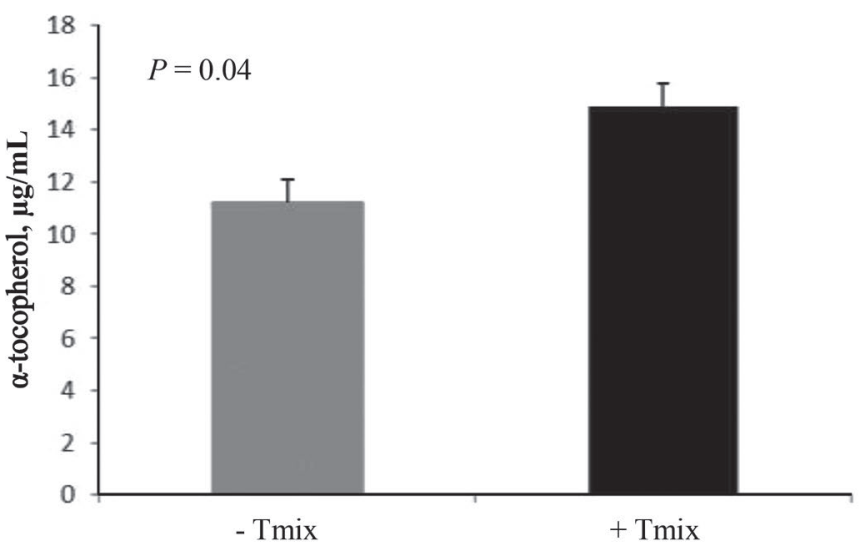

B

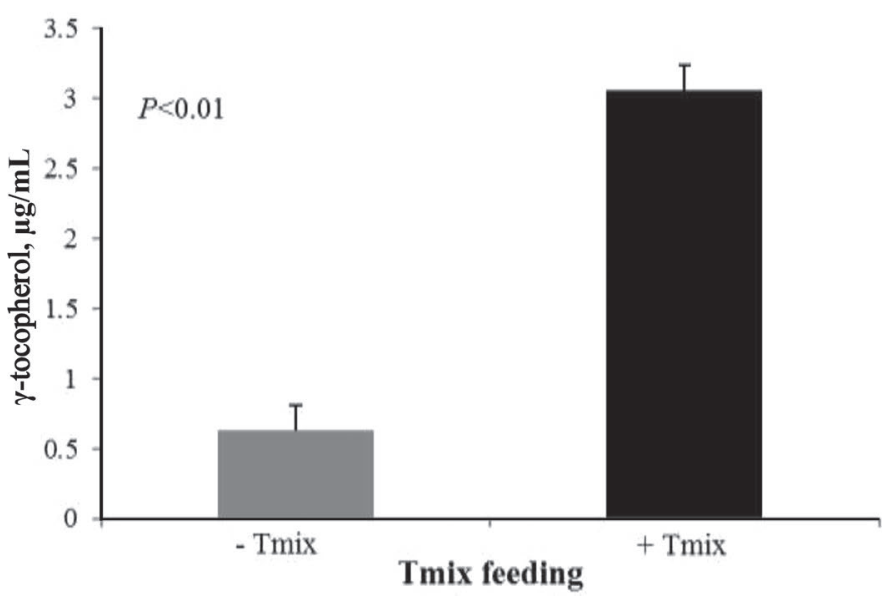

Figure 1. Tocopherol isoform measurements with (+Tmix) and without (-Tmix) feeding a mixed tocopherol oil (Tmix) in bovine plasma for 12 Holstein cows during mid lactation. Error bars represent SEM.

Table 2. Concentrations of glucose $(P=0.77)$, NEFA $(P=0.17)$, and insulin $(P=0.43)$ were not changed by Tmix feeding. However, concentrations of cholesterol were decreased $(P=0.04)$ by $4 \%$ after $7 \mathrm{~d}$ of Tmix feeding.

Table 2. Blood concentrations of glucose, insulin, nonesterified fatty acids (NEFA), and cholesterol

\begin{tabular}{|c|c|c|c|c|}
\hline \multirow[b]{2}{*}{ Item } & \multicolumn{2}{|c|}{$\operatorname{Tmix}^{1}$ feeding } & \multirow[b]{2}{*}{ SEM } & \multirow[b]{2}{*}{$P$-value } \\
\hline & - Tmix & $+\operatorname{Tmix}$ & & \\
\hline Glucose, $\mathrm{mg} / \mathrm{dL}$ & 68.92 & 68.27 & 1.34 & 0.77 \\
\hline Insulin, $\mathrm{ng} / \mathrm{mL}$ & 0.23 & 0.31 & 0.04 & 0.43 \\
\hline NEFA, mEq/L & 0.42 & 0.27 & 0.06 & 0.17 \\
\hline Cholesterol, mg/dL & 392.03 & 375.63 & 13.84 & 0.04 \\
\hline
\end{tabular}

${ }^{1}$ Mixed tocopherol oil (Tmix): $300 \mathrm{~g}$ of pure tocopherol (9\% $\alpha$-, $1 \% \beta-, 24 \% \delta$-, and $62 \% \gamma$-tocopherol) was mixed with $22.68 \mathrm{~kg}$ of calf grower (Farmers Cooperative Association Inc., Frederick, MD) and then fed to all cows at $\sim 620 \mathrm{~g} / \mathrm{d}$ for 7 consecutive days. 
Table 3. Whole-blood leukocyte population profiles of Holstein cows $(\mathrm{n}=12)$ with and without feeding a Tmix during mid lactation

\begin{tabular}{|c|c|c|c|c|}
\hline \multirow[b]{2}{*}{ Cell population } & \multicolumn{2}{|c|}{ Tmix feeding } & \multirow[b]{2}{*}{ SEM } & \multirow[b]{2}{*}{$P$-value } \\
\hline & - Tmix & + Tmix & & \\
\hline$\overline{\text { Basophils, } \times 10^{3} \text { cells } / \mu \mathrm{L}}$ & 0.01 & 0.01 & 0.01 & 0.51 \\
\hline Eosinophils, $\times 10^{3}$ cells $/ \mu \mathrm{L}$ & 0.35 & 0.36 & 0.06 & 0.88 \\
\hline Lymphocytes, $\times 10^{3}$ cells $/ \mu \mathrm{L}$ & 4.00 & 4.20 & 0.23 & 0.38 \\
\hline Monocytes, $\times 10^{3}$ cells $/ \mu \mathrm{L}$ & 0.83 & 0.93 & 0.05 & 0.11 \\
\hline Neutrophils, $\times 10^{3}$ cells $/ \mu \mathrm{L}$ & 2.80 & 3.28 & 0.18 & 0.31 \\
\hline Total leukocytes, $\times 10^{3}$ cells $/ \mu \mathrm{L}$ & 8.00 & 8.78 & 0.42 & 0.34 \\
\hline
\end{tabular}

${ }^{1}$ Mixed tocopherol oil (Tmix): $300 \mathrm{~g}$ of pure tocopherol $(9 \% \alpha-, 1 \% \beta-, 24 \% \delta$-, and $62 \% \gamma$-tocopherol) was mixed with $22.68 \mathrm{~kg}$ of calf grower (Farmers Cooperative Association Inc., Frederick, MD) and then fed to all cows at $\sim 620 \mathrm{~g} / \mathrm{d}$ for 7 consecutive days.

\section{Leukocyte Population}

The leukocyte population and composition before and after Tmix supplementation are shown in Table 3. Concentrations of total leukocytes were not changed $(P$ $=0.34$ ) after Tmix feeding. Concentrations of neutrophils $(P=0.31)$, monocytes $(P=0.11)$, lymphocytes $(P=0.38)$, basophils $(P=0.51)$, and eosinophils $(P=$ $0.88)$ were not changed after Tmix feeding.

\section{Whole-Blood Respiratory Burst by Chemiluminescence}

All blood preparations responded with a respiratory burst in the presence of PMA. Supplementing Tmix did not alter $(P=0.87)$ this respiratory burst of reacting cells in whole blood (i.e., indicating the PMN respiratory burst functions) as determined by relative chemiluminescence response.

\section{PMN Gene Expression}

The effects of Tmix supplementation and LPS challenge on PMN gene expression are shown in Table 4. There was no interaction $(P>0.1)$ between LPS challenge and Tmix supplementation, which indicates that Tmix had no effect on PMN function in response to LPS. The Tmix did not affect the expression of genes coding for the inflammatory signaling pathway of bovine PMN (TLR 4, $P=0.44 ;$ TLR2, $P=0.62 ;$ PTGS2, $P=0.63)$, except for $M Y D 88$ (1.42-fold; $P<0.01)$ and NFKB1 (1.30-fold; $P=0.03$ ). The Tmix did not affect the expression of genes coding for the cytokines (IL6, $P=0.81 ;$ IL10, $P=0.68 ;$ TNFA, $P=0.11$ ). The Tmix supplementation did not affect the expression genes coding for adhesion/receptors (SELL, $P=$ $0.48 ;$ ITGB2, $P=0.77)$. In addition, Tmix did not affect $(P=0.77)$ the expression of PLAUR coding for receptors specifically reacting with tocopherol to improve PMN chemotaxis function. The Tmix increased the expression of NCF1 (1.35-fold; $P<0.01)$ and SOD2 (1.36-fold; $P=0.05)$ coding for the superoxide enzymes during PMN activation. The Tmix did not affect $(P$ $=0.68$ ) the expression of $C A S P 3$ coding for proteins involved in cell signaling pathways; it also did not affect the expression of PPARA $(P=0.85), R X R A(P=$ $0.61)$, or $R X R B(P=0.81)$ but tended to increase the expression of $R A R A$ (1.26-fold; $P=0.06$ ).

The LPS challenge increased the expression of genes coding for inflammatory signaling pathway [NFKB1 (1.70-fold; $P<0.01$ ), PTGS2 (5.20-fold; $P<0.01$ ), TLR2 (1.44-fold; $P=0.05$ ), and TLR4 (1.46-fold; $P<$ $0.01)$ ] but not others. The LPS challenge increased the expression of cytokines [TNFA (4.36-fold; $P<0.01)$, IL6 (2.78-fold; $P<0.01)$, and IL10 (2.17-fold; $P<0.01)]$. Lipopolysaccharide challenge increased the expression of PLAUR (1.42-fold; $P=0.04)$, which encodes the cellular receptor molecule involved in cell interactions of neutrophils. However, it did not affect the expression of other adhesion or receptors $(S E L L, P=0.78$; ITGB2, $P=0.77)$ of PMN. Lipopolysaccharide challenge increased the expression of NOS2 (2.35-fold; $P<$ 0.01 ) and SOD2 (1.40-fold; $P=0.04$ ), whereas LPS did not affect $(P>0.1)$ the expression of other genes (i.e., NCF1 and NCF2) coding for superoxide enzymes of PMN. Lipopolysaccharide challenge did not affect the expression of CASP3 $(P=0.68)$, coding for proteins involved in cell signaling pathways. Lipopolysaccharide challenge did not affect the expression of PPARA $(P=$ $0.82), R X R A(P=0.88), R X R B(P=0.78)$, or $R A R A$ $(P=0.87)$, coding for transcription factors involved in tocopherol-related metabolism in bovine PMN.

\section{DISCUSSION}

Very low and stable concentrations of $\beta$ - and $\delta$-tocopherol $(<0.05 \mu \mathrm{g} / \mathrm{mL})$ were observed due to low amounts of those 2 isoforms in the standard TMR diet and Tmix supplements or could be partly explained by the low absorption and fast metabolic clearance of 
these isoforms in animals (Traber, 2013). Therefore, further small intestine (i.e., duodenum and ileum) sample collections would be beneficial to better characterize isoform levels in animals across more tissue compartments. General biomarkers of metabolism, such as glucose, NEFA, and insulin, showed similar results to previous studies indicating that all cows were metabolically healthy and that Tmix feeding had minimal if any effects on the blood metabolic profile ( $\mathrm{Qu}$ et al., 2013; Garcia et al., 2016). The Tmix feeding decreased the concentrations of plasma cholesterol, where plasma cholesterol is considered a biomarker of $\alpha$-tocopherol status in the blood (Qu et al., 2013). Supplementing $\alpha$-tocopherol increased the plasma cholesterol according to a previous report (Weiss and Wyatt, 2003). The decrease in this study could partly be explained by the existence of highly enriched non- $\alpha$ tocopherol in supplements. Even though the concentration of cholesterol was decreased, the concentration of $\alpha$-tocopherol was not impaired in the study. The Tmix did not change profiles of major immune cells, and this may indicate that Tmix did not impair normal leukocyte populations, which are needed to maintain animal health during the lactation period.

The Tmix did not alter the whole-blood respiratory burst response (i.e., indicating the PMN respiratory burst functions), and this could be partly attributed to the existence of mixed tocopherol isoforms (i.e., $\beta-$, $\gamma$-, and $\delta$-tocopherol) in the Tmix supplement. In vitro studies in humans have shown that pure $\alpha$-tocopherols serve as antioxidants and inhibit the superoxide molecules in PMA-stimulated isolated PMN via regulating protein kinase C enzyme activity (Varga et al., 2008). However, the present study did not show that Tmix inhibited respiratory burst responses in whole blood. This may be partly attributed to (1) the short-term

Table 4. Effect of feeding a Tmix ${ }^{1}$ supplement and LPS challenge ${ }^{2}$ on the expression of genes from bovine blood polymorphonuclear leukocytes from Holstein cows during mid lactation $(n=6 / \text { treatment })^{3}$

\begin{tabular}{|c|c|c|c|c|}
\hline \multirow[b]{2}{*}{ Item } & \multicolumn{2}{|c|}{ Tmix } & \multicolumn{2}{|c|}{ LPS } \\
\hline & $\mathrm{FC}^{4}$ & $P$-value & $\mathrm{FC}^{5}$ & $P$-value \\
\hline \multicolumn{5}{|c|}{ Inflammatory signaling pathway genes } \\
\hline COX2 (PTGS-2) & 1.16 & 0.63 & 5.20 & $<0.01$ \\
\hline$M Y D 88$ & 1.42 & $<0.01$ & 1.14 & 0.17 \\
\hline NFKB1 & 1.30 & 0.03 & 1.70 & $<0.01$ \\
\hline TLR2 & 1.23 & 0.62 & 1.44 & 0.05 \\
\hline TLR4 & 0.91 & 0.44 & 1.46 & $<0.01$ \\
\hline \multicolumn{5}{|l|}{ Cytokines } \\
\hline IL6 & 1.06 & 0.81 & 2.78 & $<0.01$ \\
\hline IL10 & 1.11 & 0.68 & 2.17 & $<0.01$ \\
\hline TNFA & 1.52 & 0.11 & 4.36 & $<0.01$ \\
\hline \multicolumn{5}{|c|}{ Adhesion/receptor genes } \\
\hline ITGB2 & 1.03 & 0.77 & 1.03 & 0.77 \\
\hline PLAUR & 0.94 & 0.77 & 1.42 & 0.04 \\
\hline$S E L L$ & 1.15 & 0.48 & 0.95 & 0.78 \\
\hline \multicolumn{5}{|c|}{ Superoxide enzyme genes } \\
\hline NCF1 & 1.35 & $<0.01$ & 1.07 & 0.61 \\
\hline NCF2 & 1.12 & 0.59 & 0.87 & 0.48 \\
\hline NOS2 & 0.89 & 0.63 & 2.35 & $<0.01$ \\
\hline SOD2 & 1.36 & 0.05 & 1.40 & 0.04 \\
\hline \multicolumn{5}{|l|}{ Cell apoptosis genes } \\
\hline CASP3 & 1.09 & 0.68 & 1.08 & 0.68 \\
\hline \multicolumn{5}{|c|}{ Transcription factor genes } \\
\hline PPARA & 1.05 & 0.85 & 0.95 & 0.82 \\
\hline$R A R A$ & 1.26 & 0.06 & 0.98 & 0.87 \\
\hline$R X R A$ & 1.11 & 0.61 & 0.98 & 0.88 \\
\hline$R X R B$ & 1.04 & 0.81 & 1.05 & 0.78 \\
\hline
\end{tabular}

${ }^{1}$ Mixed tocopherol oil (Tmix): $300 \mathrm{~g}$ of pure tocopherol $(9 \% \alpha-, 1 \% \beta-, 24 \% \delta$-, and $62 \% \gamma$-tocopherol) was mixed with $22.68 \mathrm{~kg}$ of calf grower (Farmers Cooperative Association Inc., Frederick, MD) and then fed to all cows at $\sim 620 \mathrm{~g} / \mathrm{d}$ for 7 consecutive days.

${ }^{2}$ On d 0 and 7 , bovine blood polymorphonuclear leukocytes were isolated from dairy cows fed mixed tocopherol oil (Tmix) supplement and incubated with or without LPS $(1.5 \mu \mathrm{g} / \mathrm{mL})$ for $2 \mathrm{~h}$ in vitro.

${ }^{3}$ Data were analyzed using the $2^{-\Delta \Delta \mathrm{Ct}}$ method and presented as fold change (FC). Fold change was calculated as $\Delta \mathrm{Cq}(\mathrm{Cq}=$ cycle quantification) of a factor of interest (with Tmix or LPS) subtracted from the $\Delta \mathrm{Cq}$ of the other referential factor (without Tmix or LPS) to generate the $\Delta \Delta$ Cq value, which was linearized to a foldchange value with the formula $2^{-\Delta \Delta \mathrm{Cq}}$

${ }^{4} \mathrm{FC}=+$ Tmix $/-$ Tmix.

${ }^{5} \mathrm{FC}=+\mathrm{LPS} /-\mathrm{LPS}$ 
feeding of Tmix, (2) the physiological state of the animal (e.g., multiparous stage, mid lactation), or (3) the fact that the benefit of feeding vitamin $\mathrm{E}$ on respiratory burst response is primarily attributed to the $\alpha$-isoform (Cachia et al., 1998; Mehrzad et al., 2001). Further studies need to be conducted to compare the effects of Tmix and the effects of individual tocopherol isoforms on neutrophil respiratory burst responses from both whole blood and isolated PMN.

The increased expression of $M Y D 88$ might be more associated with the increased expression of NFKB1 than TLR in the current study. Contrary to the previous data that $\alpha$-tocopherol decreased the expression of NFKB1 in rat immune cell lines in vitro during inflammatory responses via either LPS or TNF- $\alpha$ incubation (Li-Weber et al., 2002), the current data showed opposite results. One reason could be the more significant antioxidant ability of $\alpha$-tocopherol, which reduces the production of superoxide anions that can induce the proinflammatory responses via nuclear factor $\kappa \mathrm{B}$ signaling pathways compared with other isoforms (Conner and Grisham, 1996). Besides $\alpha$-tocopherol, $\gamma$-tocopherol displays anti-inflammatory capabilities by serving as an antioxidant by scavenge nitrogen dioxide, thereby reducing the expression of PTGS2 (Campbell et al., 2003). In the current study, Tmix (highly enriched with $\gamma$-tocopherol) did not alter the expression of PTGS2, an enzyme responsible for formation of prostanoids during inflammation (Williams et al., 1999). It is possible that the tocopherol-metabolized compound (i.e., carboxyethyl-hydroxychromans) showed more direct inhibition effects in expression of PTGS2 compared with tocopherols (Egger et al., 2003; Grammas et al., 2004). Ultimately, compared with previous studies, the present results showed different effects of tocopherol on the expression of proinflammatory genes (i.e., NFKB1 and $M Y D 88)$. A possible explanation is the fact that the Tmix comprises all 4 isoforms where the $\beta$ - or $\delta$-tocopherol isoforms may have interacted with the $\alpha$ or $\gamma$-tocopherol isoforms in proinflammatory pathways. Even though Tmix increased the expression of genes involved in proinflammatory responses, it does not indicate that high expression of those genes is harmful to blood PMN. The increased expression of proinflammatory genes might be necessary during the initiation of innate immune functions and later activation of adaptive immune functions (Lin and Karin, 2007). Further studies are needed to compare the in vitro effects of Tmix with individual $\alpha$ - or $\gamma$-tocopherols on the PMN expression of proinflammatory genes.

The Tmix did not alter the expression of adhesion/ receptor genes (PLAUR, ITGB2, and SELL), indicating that Tmix may not affect other PMN functions after short-term feeding during mid lactation. Furthermore,
Tmix did not alter the expression of TLR4 and TLR2, both of which have been shown to increase during the inflammatory response (Moyes et al., 2010; Ingvartsen and Moyes, 2015). It was expected that Tmix would depress the expression of TLR4 or TLR2 to inhibit the proinflammatory responses induced by LPS challenge. The results did not show changes in the expression of these 2 genes, which might indicate that tocopherol did not interact with PMN surface receptors in regulating the inflammation reaction in PMN during LPS challenge even though tocopherol is located mainly in the cell membrane (Li and May, 2003). This needs to be confirmed via an in vitro incubation experiment.

The Tmix increased the expression of SOD2, an enzyme used to convert superoxide molecules to hydrogen peroxide when PMN are activated via LPS challenge. Our results are similar to those reported by others (Saldeen et al., 1999; Salvesen et al., 2016). The present data also showed that Tmix increased the expression of NCF1 coding for the subunit of NADPH oxidase to produce superoxide molecules in activated PMN. This might partly explain the above chemiluminescence data wherein Tmix did not alter the respiratory burst responses of whole blood, possibly due to interactions with the other tocopherol isoforms (i.e., $\beta$ - and $\delta$-tocopherol). In addition, Tmix showed no effect on the expression of $C A S P 3$ coding mediators involved in cell apoptosis pathways

Furthermore, Tmix did not alter the expression of transcription factor genes (i.e., PPARA, RXRA, or $R X R B)$. According to previous studies, tocopherol serves as a ligand to bind with PPARA paired with $R X R$ to initiate tocopherol metabolism pathways in mammals during tocopherol supplementation to maintain the stable level of tocopherol in the body (Azzi et al., 2004; Traber, 2004). This may indicate that the $\alpha$-isoform may play a vital role in transcription when compared with other isoforms. Supplementing Tmix tended to increase the expression of $R A R A$ and might be partly attributed to the negative interaction of retinoic acid and tocopherol, where tocopherol could stimulate the metabolism of retinoic acid (Schelling et al., 1995). Overall, the current data did not demonstrate any major changes in blood PMN gene expression associated with Tmix feeding despite the major role that PMN play in the innate immune response to microorganism invasion.

\section{CONCLUSIONS}

In conclusion, under the conditions of this study, Tmix supplementation did not have a harmful effect on animal health. Our results show that feeding Tmix increased $\alpha$ - and $\gamma$-tocopherol concentrations 
in blood, did not impair whole-blood cellular respiratory burst activity or alter leukocyte populations, and did not affect the concentration of major biomarkers of metabolism (plasma glucose, NEFA, and insulin). Gene expression profiles of PMN stimulated or not stimulated with LPS indicated that supplementation of Tmix differentially affected genes associated with the proinflammatory response cascade. These observations warrant further study and may prove that adding Tmix to the diet could improve some parameters of the immune response.

\section{ACKNOWLEDGMENTS}

This study was funded by the Maryland Agricultural Experiment Station and the USDA Agricultural Research Service (ARS) in-house funding (CRIS no. 804231320-077-00D). The authors acknowledge the dairy group from the USDA ARS Beltsville Agricultural Research Center (Beltsville, MD) for their assistance with animal care and data collection. The authors also acknowledge Neal Craft and the staff from Craft Technologies Inc. (Wilson, NC) for the HPLC analysis and Matthew Kramer from the statistics group (USDA ARS Northeast Area, Beltsville, MD) for statistics consultation.

\section{REFERENCES}

Akira, S., and H. Hemmi. 2003. Recognition of pathogen-associated molecular patterns by TLR family. Immunol. Lett. 85:85-95.

Azzi, A., R. Gysin, P. Kempna, A. Munteanu, Y. Negis, L. Villacorta, T. Visarius, and J. M. Zingg. 2004. Vitamin E mediates cell signaling and regulation of gene expression. Ann. N. Y. Acad. Sci. 1031:86-95. https://doi.org/10.1196/annals.1331.009.

Barrett, J. J., J. S. Hogan, W. P. Weiss, K. L. Smith, and L. M. Sordillo. 1997. Concentrations of alpha-tocopherol after intramammary infusion of Escherichia coli or lipopolysaccharide. J. Dairy Sci. 80:2826-2832. https://doi.org/10.3168/jds.S0022-0302(97)76247 -7 .

Benjamini, Y., and Y. Hochberg. 1995. Controlling the false discovery rate: A practical and powerful approach to multiple testing. J. R. Stat. Soc. Series B Stat. Methodol. 57:289-300.

Bionaz, M., E. Trevisi, L. Calamari, F. Librandi, A. Ferrari, and G. Bertoni. 2007. Plasma paraoxonase, health, inflammatory conditions, and liver function in transition dairy cows. J. Dairy Sci. 90:1740-1750. https://doi.org/10.3168/jds.2006-445.

Blum, J. W., H. Dosogne, D. Hoeben, F. Vangroenweghe, H. M. Hammon, R. M. Bruckmaier, and C. Burvenich. 2000. Tumor necrosis factor-alpha and nitrite/nitrate responses during acute mastitis induced by Escherichia coli infection and endotoxin in dairy cows. Domest. Anim. Endocrinol. 19:223-235. https://doi.org/10.1016/ S0739-7240(00)00079-5.

Cachia, O., J. El Benna, E. Pedruzzi, B. Descomps, M. A. GougerotPocidalo, and C. L. Leger. 1998. Alpha-tocopherol inhibits the respiratory burst in human monocytes attenuation of $\mathrm{p} 47$ phox membrane translocation and phosphorylation. J. Biol. Chem. 273:32801-32805.

Campbell, S. E., W. L. Stone, S. G. Whaley, M. Qui, and K. Krishnan. 2003. Gamma (gamma) tocopherol upregulates peroxisome proliferator activated receptor (PPAR) gamma (gamma) expression in SW 480 human colon cancer cell lines. BMC Cancer 3:25-38. https://doi.org/10.1186/1471-2407-3-25.

Chew, B. P. 1995. Antioxidant vitamins affect food animal immunity and health. J. Nutr. 125:1804S-1808S.

Conner, E. M., and M. B. Grisham. 1996. Inflammation, free radicals, and antioxidants. Nutrition 12:274-277. https://doi.org/10.1016/ S0899-9007(96)00000-8.

Drackley, J. K., S. S. Donkin, and C. K. Reynolds. 2006. Major advance in fundamental dairy cattle nutrition. J. Dairy Sci. 89:13241336. https://doi.org/10.3168/jds.S0022-0302(06)72200-7.

Egger, T., R. Schuligoi, A. Wintersperger, R. Amann, E. Malle, and W. Sattler. 2003. Vitamin E (alpha-tocopherol) attenuates cyclooxygenase 2 transcription and synthesis in immortalized murine BV-2 microglia. Biochem. J. 370:459-467. https://doi.org/10 .1042/BJ20021358.

Elsasser, T. H., S. Kahl, K. M. Lebold, M. G. Traber, J. Shaffer, C. Li, and S. Block. 2013. Short-term alpha-or gamma-delta-enriched tocopherol oil supplementation differentially effects the expression of proinflammatory mediators: Selective impacts on characteristics of protein tyrosine nitration in vivo. Vet. Sci Dev. 3:20-38. https:/ doi.org/10.4081/vsd.2013.4703.

Ferguson, J. D.. D. T. Galligan, and N. Thomsen. 1994. Principal descriptors of body condition score in Holstein cows. J. Dairy Sci. 77:2695-2703. https://doi.org/10.3168/jds.S0022-0302(94)77212 $-\mathrm{X}$.

Freshney, R. 1987. Culture of Animal Cells: A Manual of Basic Technique. Liss, New York, NY.

Garcia, M., T. H. Elsasser, D. Biswas, and K. M. Moyes. 2015a. The effect of citrus-derived oil on bovine blood neutrophil function and gene expression in vitro. J. Dairy Sci. 98:918-926. https://doi.org/ $10.3168 /$ jds.2014-8450.

Garcia, M., T. H. Elsasser, L. Juengst, Y. Qu, B. J. Bequette, and K. M. Moyes. 2016. Short communication: Amino acid supplementation and stage of lactation alter apparent utilization of nutrients by blood neutrophils from lactating dairy cows in vitro. J. Dairy Sci. 99:3777-3783. https://doi.org/10.3168/jds.2015-10086.

Garcia, M., T. H. Elsasser, Y. Qu, X. Zhu, and K. M. Moyes. 2015b. Glucose supplementation has minimal effects on blood neutrophil function and gene expression in vitro. J. Dairy Sci. 98:6139-6150. https://doi.org/10.3168/jds.2014-9183.

Grammas, P., L. Hamdheydari, E. J. Benaksas, S. Mou, Q. N. Pye, W. J. Wechter, R. A. Floyd, C. Stewart, and K. Hensley. 2004. Anti-inflammatory effects of tocopherol metabolites. Biochem. Biophys. Res. Commun. 319:1047-1052. https://doi.org/10.1016/ j.bbrc.2004.05.082.

Groeger, A. L., and B. A. Freeman. 2010. Signaling actions of electrophiles: Anti-inflammatory therapeutic candidates. Mol. Interv. 10:39-50. https://doi.org/10.1038/nchembio.367.

Gruet, P., P. Maincent, X. Berthelot, and V. Kaltsatos. 2001. Bovine mastitis and intramammary drug delivery: Review and perspectives. Adv. Drug Deliv. Rev. 50:245-259. https://doi.org/10.1016/ S0169-409X(01)00160-0.

Higuchi, H., E. Ito, H. Iwano, S. Oikawa, and H. Nagahata. 2013. Effects of vitamin E supplementation on cellular alpha-tocopherol concentrations of neutrophils in Holstein calves. Can. J. Vet. Res. $77: 120-125$.

Hogan, J. S., W. P. Weiss, D. A. Todhunter, K. L. Smith, and P. S. Schoenberger. 1992. Bovine neutrophil responses to parenteral vitamin E. J. Dairy Sci. 75:399-405. https://doi.org/10.3168/jds .S0022-0302(92)77775-3.

Ingvartsen, K. L., and K. M. Moyes. 2015. Factors contributing to immunosuppression in the dairy cow during the periparturient period. Jpn. J. Vet. Res. 63(Suppl. 1):15-24. http://hdl.handle.net/ $2115 / 57936$

Lauzon, K., X. Zhao, A. Bouetard, L. Delbecchi, B. Paquette, and P. Lacasse. 2005. Antioxidants to prevent bovine neutrophil-induced mammary epithelial cell damage. J. Dairy Sci. 88:4295-4303. https://doi.org/10.3168/jds.S0022-0302(05)73115-5.

Li, X., and J. M. May. 2003. Location and recycling of mitochondrial alpha-tocopherol. Mitochondrion 3:29-38. https://doi.org/10 .1016/S1567-7249(03)00058-8. 
Li-Weber, M., M. Giaisi, M. K. Treiber, and P. H. Krammer. 2002. Vitamin E inhibits IL-4 gene expression in peripheral blood T cells. Eur. J. Immunol. 32:2401-2408. https://doi.org/10.1002/ 1521-4141(200209)32:9<2401::AID-IMMU2401>3.0.CO:2-S.

Lieberman, M. M., D. M. Sachanandani, and C. A. Pinney. 1996. Comparative study of neutrophil activation by chemiluminescence and flow cytometry. Clin. Diagn. Lab. Immunol. 3:654-662.

Lin, W. W., and M. Karin. 2007. A cytokine-mediated link between innate immunity, inflammation, and cancer. J. Clin. Invest. 117:1175-1183. https://doi.org/10.1172/JCI31537.

Livak, K. J., and T. D. Schmittgen. 2001. Analysis of relative gene expression data using real-time quantitative PCR and the 2(-Delta Delta C(T)). Methods 25:402-408. https://doi.org/10.1006/meth .2001 .1262

Luostarinen, R., A. Siegbahn, and T. Saldeen. 1991. Effects of dietary supplementation with vitamin $\mathrm{E}$ on human neutrophil chemotaxis and generation of LTB4. Ups. J. Med. Sci. 96:103-111.

Mehrzad, J., H. Dosogne, E. Meyer, R. Heyneman, and C. Burvenich. 2001. Respiratory burst activity of blood and milk neutrophils in dairy cows during different stage of lactation. J. Dairy Res. 68:399-415. https://doi.org/10.1017/S0022029901005039.

Mehrzad, J., L. Duchateau, S. Pyorala, and C. Burvenich. 2002. Blood and milk neutrophil chemiluminescence and viability in primiparous and pluriparous dairy cows during late pregnancy, around parturition and early lactation. J. Dairy Sci. 85:3268-3276. https://doi.org/10.3168/jds.S0022-0302(02)74415-9.

Moyes, K. M., J. K. Drackley, D. E. Morin, and J. J. Loor. 2010 Greater expression of TLR2, TLR4, and IL6 due to negative energy balance is associated with lower expression of HLA-DRA and HLA-A in bovine blood neutrophils after intramammary mastitis challenge with Streptococcus uberis. Funct. Integr. Genomics 10:53-61. https://doi.org/10.1007/s10142-009-0154-7.

NRC (National Research Council). 2001. Nutrient Requirements of Dairy Cattle. 7th rev. ed. Natl. Acad. Press, Washington, DC.

Netscher, T., F. Mazzini, and R. Jestin. 2007. Tocopherols by hydride reduction of dialkylamino derivatives. Eur. J. Org. Chem. 7:11761183. https://doi.org/10.1002/ejoc.200600874.

Paape, M. J., D. D. Bannerman, X. Zhao, and J. W. Lee. 2003. The bovine neutrophil: Structure and function in blood and milk. Vet. Res. 34:597-627. https://doi.org/10.1051/vetres:2003024.

Persson, K., I. Larsson, and S. C. Hallen. 1993. Effects of certain inflammatory mediators on bovine neutrophil migration in vivo and in vitro. Vet. Immunol. Immunopathol. 37:99-112. https://doi .org/10.1016/0165-2427(93)90058-C.

Pilotto, A., G. Savoini, A. Baldi, G. Invernizzi, V. C. De, G. Theodorou, P. Koutsouli, and I. Politis. 2016. Short communication: Associations between blood fatty acids, beta-hydroxybutyrate, and alpha-tocopherol in the periparturient period in dairy cows: An observational study. J. Dairy Sci. 99:8121-8126. https://doi.org/ 10.3168/jds.2016-10938.

Pinotti, L., R. Rebucci, E. Fusi, L. Rossi, and A. Baldi. 2003. Milk choline, alpha-tocopherol and neutrophil chemotaxis in the periparturient dairy cow. Vet. Res. Commun. 27(Suppl. 1):265-268.

Politis, I., N. Hidiroglou, J. H. White, J. A. Gilmore, S. N. Williams, H. Scherf, and M. Frigg. 1996. Effects of vitamin E on mammary and blood leukocyte function, with emphasis on chemotaxis, in periparturient dairy cows. Am. J. Vet. Res. 57:468-471.

Politis, I., G. Theodorou, A. D. Lampidonis, A. Kominakis, and A. Baldi. 2012. Short communication: Oxidative status and incidence of mastitis relative to blood alpha-tocopherol concentrations in the postpartum period in dairy cows. J. Dairy Sci. 95:7331-7335. https://doi.org/10.3168/jds.2012-5866.

Qu, Y., A. N. Fadden, M. G. Traber, and G. Bobe. 2014. Potential risk indicators of retained placenta and other diseases in multiparous cows. J. Dairy Sci. 97:4151-4165. https://doi.org/10.3168/jds.2013 -7154 .

Qu, Y., K. Lytle, M. G. Traber, and G. Bobe. 2013. Depleted serum vitamin $\mathrm{E}$ concentrations precede left displaced abomasum in early-lactation dairy cows. J. Dairy Sci. 96:3012-3022. https://doi .org/10.3168/jds.2012-6357.

R Core Team. 2014. R: A Language and Environment for Statistical Computing. R Core Team, Vienna, Austria.

Saldeen, T., D. Li, and J. L. Mehta. 1999. Differential effects of alpha- and gamma-tocopherol on low-density lipoprotein oxidation, superoxide activity, platelet aggregation and arterial thrombogenesis. J. Am. Coll. Cardiol. 34:1208-1215. https://doi.org/10.1016/ S0735-1097(99)00333-2.

Salvesen, Ø., M. R. Reiten, P. M. H. Heegaard, M. A. Tranulis, A. Espenes, K. Skovgaard, and C. Ersdal. 2016. Activation of innate immune genes in caprine blood leukocytes after systemic endotoxin challenge. BMC Vet. Res. 12:241-251. https://doi.org/10.1186/ s12917-016-0870-x.

Schelling, G. T., R. A. Roeder, M. J. Garber, and W. M. Pumfrey. 1995. Bioavailability and interaction of vitamin A and vitamin E in ruminants. J. Nutr. 125:1799S-1803S.

Singh, A. K., S. Pandita, Z. A. Pampoori, R. Huozha, and M. Ashutosh. 2013. Effect of pre-partum supplementation of vitamin $\mathrm{E}$ to Murrah buffaloes on immune functions and viability of calves. J. Anim. Physiol. Anim. Nutr. (Berl.) 97:255-262. https://doi.org/ 10.1111/j.1439-0396.2011.01264.x.

Sordillo, L. M. 2016. Nutritional strategies to optimize dairy cattle immunity. J. Dairy Sci. 99:4967-4982. https://doi.org/10.3168/jds .2015-10354

Sordillo, L. M., G. A. Contreras, and S. L. Aitken. 2009. Metabolic factors affecting the inflammatory response of periparturient dairy cows. Anim. Health Res. Rev. 10:53-63. https://doi.org/10.1017/ S1466252309990016.

Spears, J. W., and W. P. Weiss. 2008. Role of antioxidants and trace elements in health and immunity of transition dairy cows. Vet. J. 176:70-76. https://doi.org/10.1016/j.tvjl.2007.12.015.

Tian, S. Z., C. J. Chang, C. C. Chiang, H. C. Peh, M. C. Huang, J. W. Lee, and X. Zhao. 2005. Comparison of morphology, viability, and function between blood and milk neutrophils from peak lactating goats. Can. J. Vet. Res. 69:39-45.

Traber, M. G. 2004. Vitamin E, nuclear receptors and xenobiotic metabolism. Arch. Biochem. Biophys. 423:6-11. https://doi.org/10 .1016/j.abb.2003.10.009.

Traber, M. G. 2013. Mechanisms for the prevention of vitamin E excess. J. Lipid Res. 54:2295-2306. https://doi.org/10.1194/jlr .R032946.

Varga, Z., E. Kosaras, E. Komodi, M. Katko, I. Karpati, J. Balla, G. Paragh, M. C. Aisa, and F. Galli. 2008. Effects of tocopherols and $2,2^{\prime}$-carboxyethyl hydroxychromans on phorbol-ester-stimulated neutrophils. J. Nutr. Biochem. 19:320-327. https://doi.org/10 .1016/j.jnutbio.2007.05.002.

Weiss, W. P., J. S. Hogan, D. A. Todhunter, and K. L. Smith. 1997. Effect of vitamin E supplementation in diets with a low concentration of selenium on mammary gland health of dairy cows. J. Dairy Sci. 80:1728-1737. https://doi.org/10.3168/jds.S0022 -0302(97)76105-8.

Weiss, W. P., and N. R. St-Pierre. 2009. A method to quantify changes in supply of metabolizable methionine to dairy cows using concentrations of selenium in milk. J. Dairy Sci. 92:2835-2842. https:// doi.org/10.3168/jds.2008-1882.

Weiss, W. P., and D. J. Wyatt. 2003. Effect of dietary fat and vitamin E on alpha-tocopherol in milk from dairy cows. J. Dairy Sci 86:3582-3591. https://doi.org/10.3168/jds.S0022-0302(03)73964 -2 .

Wellnitz, O., and R. M. Bruckmaier. 2012. The innate immune response of the bovine mammary gland to bacterial infection. Vet. J. 192:148-152. https://doi.org/10.1016/j.tvjl.2011.09.013.

Williams, C. S., M. Mann, and R. N. Dubois. 1999. The role of cyclooxygenases in inflammation, cancer, and development. Oncogene 18:7908-7916.

Zempleni, J., J. W. Suttle, J. F. Gregory III, and P. J. Stover. 2013. Handbooks of Vitamins. CRC Press, Boca Raton, FL. 